\title{
KCNT1-related epilepsy: An international multicenter cohort of 27 pediatric cases
}

Felippe Borlot $^{1}$ (D) | Ahmed Abushama ${ }^{1}$ | Nadine Morrison-Levy ${ }^{1,2}$ | Puneet Jain ${ }^{1,3}$ | Kollencheri Puthenveettil Vinayan ${ }^{4}$ | Musaad Abukhalid ${ }^{5}$ | Hesham M. Aldhalaan ${ }^{5}$ Hanin S. Almuzaini ${ }^{5}$ ｜ Sheffali Gulati ${ }^{6}$ ｜ Tova Hershkovitz $^{7}$ ｜ Ramesh Konanki $^{8}$ | Lokesh Lingappa $^{8}$ | Aimee F. Luat ${ }^{9} \quad$ Shatha Shafi ${ }^{10}$ ｜ Brahim Tabarki ${ }^{10}$ |

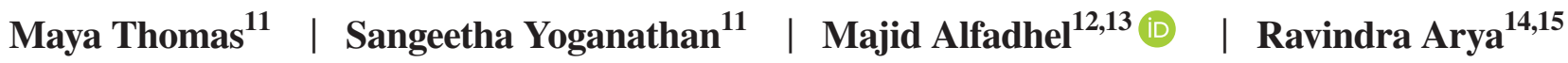
Elizabeth J. Donner $^{1}$ | Salleh N. Ehaideb ${ }^{12}$ | Vykuntaraju K. Gowda ${ }^{16}$ | Vivek Jain $^{17}$ | Priyanka Madaan ${ }^{18}$ | Kenneth A. Myers ${ }^{19,20,21}$ (D) | Hiroshi Otsubo | Prateek Panda $^{6}$ | Jitendra K. Sahu ${ }^{18}$ (iD | Letícia P. B. Sampaio ${ }^{22}$ | Suvasini Sharma ${ }^{23}$ Elisabeth Simard-Tremblay $^{19,20} \mid$ Maria Zak $^{1} \mid$ Robyn Whitney ${ }^{1}$

${ }^{1}$ Division of Neurology, Department of Paediatrics, The Hospital for Sick Children, Toronto, Ontario, Canada

${ }^{2}$ Children's Hospital of Eastern Ottawa, Ottawa, Ontario, Canada

${ }^{3}$ Division of Pediatric Neurology, Department of Pediatrics, Danat Al Emarat Hospital for Women and Children, Abu Dhabi, United Arab Emirates

${ }^{4}$ Department of Pediatric Neurology, Amrita Institute of Medical Sciences, Cochin, India

${ }^{5}$ Department of Neurosciences, King Faisal Specialist Hospital and Research Center, Riyadh, Saudi Arabia

${ }^{6}$ Child Neurology Division, Department of Pediatrics, Center of Excellence \& Advanced Research on Childhood Neurodevelopmental Disorders, All India Institute of Medical Sciences, New Delhi, India

${ }^{7}$ Genetic Institute, Rambam Medical Center, Haifa, Israel

${ }^{8}$ Department of Neurology, Rainbow Children's Hospital, Hyderabad, India

${ }^{9}$ Detroit Medical Center, Children's Hospital of Michigan, Wayne State University School of Medicine, Detroit, Michigan

${ }^{10}$ Division of Neurology, Department of Pediatrics, Prince Sultan Military Medical City, Riyadh, Saudi Arabia

${ }^{11}$ Pediatric Neurology, Department of Neurological Sciences, Christian Medical College, Vellore, India

${ }^{12}$ King Abdullah International Medical Research Center, King Saud bin Abdulaziz University for Health Sciences, King Abdulaziz Medical City, Ministry of National Guard-Health Affairs, Riyadh, Saudi Arabia

${ }^{13}$ Division of Genetics, Department of Pediatrics, King Abdulaziz Medical City, Ministry of National Guard-Health Affairs, Riyadh, Saudi Arabia

${ }^{14}$ Division of Neurology, Comprehensive Epilepsy Center, Cincinnati Children's Hospital Medical Center, Cincinnati, Ohio

${ }^{15}$ Department of Pediatrics, University of Cincinnati College of Medicine, Cincinnati, Ohio

${ }^{16}$ Indira Gandhi Institute of Child Health, Bangalore, India

${ }^{17}$ Santokba Durlabhji Hospital, Jaipur, India

${ }^{18}$ Pediatric Neurology Unit, Department of Pediatrics, Postgraduate Institute of Medical Education and Research, Chandigarh, India

${ }^{19}$ Division of Neurology, Department of Pediatrics, Montreal Children's Hospital, McGill University Health Centre, Montreal, Quebec, Canada

${ }^{20}$ Department of Neurology \& Neurosurgery, McGill University Health Centre, Montreal, Quebec, Canada

${ }^{21}$ Research Institute of the McGill University Health Centre, Montreal, Quebec, Canada

${ }^{22}$ Department of Neurology, Faculty of Medicine, University of Sao Paulo, Sao Paulo, Brazil

${ }^{23}$ Neurology Division, Department of Pediatrics, Lady Harding Medical College and Kalawati Saran Children's Hospital, New Delhi, India 


\section{Correspondence}

Robyn Whitney, The Division of Neurology, The Hospital for Sick Children, 555 University Ave, Toronto, ON M5G $1 \mathrm{X} 8$, Canada.

Email: robyn.whitney@sickkids.ca

\section{1 | INTRODUCTION}

KCNT1 encodes a ligand-gated potassium channel, which is activated by intracellular sodium binding (also called SLACK, SLO2.2, KC4.1). KCNT1 has several functions, which include regulating neuronal firing rate, contributing to the slow hyperpolarization after repetitive firing, and it also has an important role in neuronal response to hypoxia. ${ }^{1-3}$ Gain-of-function effects produce higher action potential firing frequency due to faster action potential repolarization and increased fast afterhyperpolarization. ${ }^{4} \mathrm{KCNT} 1$ channels are widely expressed in the central nervous system and are found in the olfactory bulb, brainstem, and hippocampal and cortical embryonic neurons. ${ }^{1,2,5}$

Gain-of-function $K C N T 1$ pathogenic variants are known to cause pleiotropic effects, and a number of epilepsy phenotypes have been described: (1) epilepsy of infancy with migrating focal seizures (EIMFS) ${ }^{1,6}$; (2) a severe form of autosomal dominant sleep-related hypermotor epilepsy $(\mathrm{ADSHE})^{7}$; (3) early onset epileptic encephalopathy (EOEE; ie, Ohtahara syndrome, West syndrome, and unclassified EOEE $)^{8}$; (4) temporal lobe epilepsy with intellectual disability ${ }^{9}$; and (5) myoclonic-atonic epilepsy. ${ }^{10}$ Although most patients harboring $K C N T 1$ pathogenic variants have no causative underlying structural brain abnormalities, patients 
with ADSHE may rarely exhibit malformations of cortical development. ${ }^{11}$

There have been inconsistent data with respect to clinical efficacy of quinidine (a broad-spectrum potassium channel blocker) in patients with EIMFS. Unblinded assessment of seizure reduction may range from complete to no response. ${ }^{12-17}$ Overall, half of EIMFS or EOEE patients will have no response to quinidine, and only $20 \%$ may benefit with at least a $50 \%$ seizure reduction. ${ }^{18}$ The main adverse effect attributed to quinidine therapy is cardiotoxicity with prolonged QTc interval and arrhythmias, but sedation, elevated liver function tests, rash, and skin discoloration have also been described. ${ }^{18,19}$

We sought to evaluate the clinical aspects, including phenotypic presentation, electroencephalogram (EEG), neuroimaging, and response to pharmacological and nonpharmacological therapies of a multiethnic cohort of children with pathogenic or likely pathogenic $K C N T 1$ variants. Despite the high pleiotropy and heterogeneity associated with KCNT1related epilepsies, we also aimed to explore any possible phenotype-genotype correlations associated with the most common identified variants.

\section{2 | MATERIALS AND METHODS}

\subsection{Patients and institutional review board approval}

Ethics approval for the study was granted by the Research Ethics Board at the Hospital for Sick Children, Toronto, Ontario, Canada (REB \#1000061319) and the other participant centers, as per the respective hospital policies. Children were enrolled in the study after consent was obtained from legal guardians, unless the local board institution waived the need to obtain informed consent from a given center. Recruitment started within the Division of Neurology at the Hospital for Sick Children, which was the coordinating research site. Henceforth, an international collaborative network joined the study, allowing us to recruit patients from Brazil, Canada, India, Israel, Saudi Arabia, and the USA. All mutations were identified by commercial gene panels or whole exome sequencing.

\section{2 | Study design, and inclusion and exclusion criteria}

Inclusion criteria for this retrospective case series study were (1) age 18 years or younger; (2) diagnosed with KCNT1related epilepsy at any point before September 2018; and (3) pathogenic or likely pathogenic $K C N T 1$ variants (class 5 or 4), according to the American College of Medical Genetics

\section{Key Points}

- KCNT1-related epilepsy in children usually manifests as early onset refractory focal tonic seizures and EIMFS

- Most children will become nonambulatory, nonverbal, hypotonic, and spastic, with acquired microcephaly and cortical visual impairment

- Supportive MRI findings include delayed myelination, thin corpus callosum, brain atrophy, and malformations of cortical developmental

- There have been no well-established genotypephenotype-specific correlations so far

- Despite the lack of evidence-based practice, ketogenic diet and quinidine are well tolerated and may help with seizure reduction

and Genomics (ACMG), ${ }^{20,21}$ regardless of the phenotypic presentation. The exclusion criteria were (1) harboring $K C N T 1$ variants of uncertain significance (VUS) or benign variants and (2) failure to gather enough data for phenotypic characterization.

\section{3 | Data collection, analysis, and interpretation}

Study data were collected, stored, and managed using the REDCap (Research Electronic Data Capture) secure electronic data capture tool at the Hospital for Sick Children. ${ }^{22,23}$ Data abstraction protocol included demographics, age of presentation, first symptom developed by the patient, age at seizure onset, seizure type(s), presence of known epilepsy syndrome, developmental history, neurological/psychiatric/ systemic comorbidities, genotype and inheritance (if available), EEG reports, neuroimaging abnormalities, pharmacological and nonpharmacological treatment, and treatment response. For each treatment modality, the following options were gathered from caregivers and clinicians: (1) seizure freedom, (2) marked improvement (ie, $>50 \%$ reduction in seizures), (3) some improvement (ie, 25\%-50\% seizure reduction), (4) minimal improvement (ie, $<25 \%$ seizure reduction), (5) no improvement, and (6) worsening of seizures. In addition, tolerability, adverse effects, reason for discontinuation of therapy, and duration of therapies were gathered. Data were summarized using descriptive statistics, including mean, median, range, and standard deviation (SD) for continuous variables and percentage for categorical variables.

Clinical genetic testing (ie, through gene panels or whole exome sequencing) was performed through different companies internationally, using their specific protocols to obtain 
genomic DNA from blood. Testing of family members was performed according to clinical indication and/or availability using Sanger sequencing in nearly all cases. Variants of uncertain significance, and benign and likely benign variants in other genes, as well as heterozygous pathogenic variants detected in genes associated with autosomal recessive conditions, were considered out of the scope of the study and will not be reported.

To address the second aim of the study (ie, to determine phenotype-genotype correlations associated with more frequently encountered variants), we performed the chi-square test (IBM SPSS Statistics v20.0) to compare the presence of specific findings (such as developmental plateauing, presence of EIMFS, reported seizure frequency $>500 / \mathrm{mo}$, abnormal magnetic resonance imaging [MRI]) in the most frequently encountered $K C N T 1$ variants found in our cohort (ie, 2800G > A; p.Ala934Thr and c.862G > A; p.Gly288Ser). In addition, the chi-square test was used to compare children with and without these variants, with respect to treatment responses to quinidine, diet therapy, and cannabidiol (CBD). Moreover, given the previous literature data suggesting that an earlier age at onset of quinidine therapy could result in a better response, we compared the age at initiation of quinidine therapy to the seizure reduction response using Spearman $\rho$ calculator. Statistically significant correlations were considered positive if $P<.05$.

All data were reviewed by two authors from the main research site for completeness; whenever incomplete or unclear information was noted, further clarification was sought from the contributing center. Individual genetic results (KCNTI variants) obtained from each center were checked and reclassified (if required) in accordance with the ACMG criteria. ${ }^{20,21}$ If, on reclassification, patients were found to have VUS or benign variants, they were then subsequently excluded from the analysis.

\section{$3 \mid$ RESULTS}

Thirty children were initially recruited from all centers. However, three patients were excluded upon review, as their KCNT1 variants were classified as VUS. Our cohort was composed of 27 children, 15 males and 12 females (ratio = $1.25)$, current mean age $=40.8$ months $(\mathrm{SD}=28.3$, median $=$ 38 months). Three patients were previously published (Cases $15,16$, and 25$),{ }^{24,25}$ and 24 children are newly reported. For all children, seizures were the first concern that brought them to attention of a neurologist, with age at onset of seizures ranging from 1 day to 6 months of life (mean $=1.7$ months, $\mathrm{SD}=1.7$, median $=1$ month). Nevertheless, the mean age for a definitive diagnosis of KCNT1-related epilepsy was later, at mean of 18.8 months, $\mathrm{SD}=20.1$ (median $=10$ months). Developmental trajectory was characterized by plateauing in nearly half of children $(48.1 \%, 13 / 27)$ upon seizure onset, whereas $22.2 \%(6 / 27)$ had slow developmental gains, and $22.2 \%(6 / 27)$ had developmental regression, followed by either slow gains or plateauing.

Recurrent $K C N T 1$ variants were reported in 18 patients. The most common KCNT1 variants were (1) c.2800G $>$ A; p.Ala934Thr, (2) c.862G $>$ A; p.Gly288Ser, and (3) c.1421G $>$ A; p.Arg474His. These variants were, respectively, seen in five (Cases 4, 8, 16, 24, and 27), four (Cases 7, 9, 10, and 11), and three (Cases 6, 17, and 20) children. In addition, (4) c.1283G > A; p.Arg428Gln (Cases 1 and 23), (5) c.1420C $>$ T; p.Arg474Cys (Cases 13 and 22), and (6) c.2849G >A; p.Arg950Gln (Cases 3 and 15) were found in two children each. From the nine nonrecurrent $K C N T 1$ variants found in this cohort, four have not been previously reported in the literature (Cases 2, 12, 14, and 18). Case 12's nucleotide variant (c.1130G $>$ C) is novel; however, a nucleotide variant affecting the same amino acid (c.1129T $>$ A; $p$. Cys377Ser) has been reported in a patient presenting with EIMFS. ${ }^{26}$

Parental testing was completed in 24 of the 27 families, and 23 children $(95.8 \%, 23 / 24)$ were found to have de novo variants. Despite having a de novo variant, six of the 23 children $(26 \%, 6 / 23)$ with a de novo variant had a positive family history of seizures. We had only limited information on the specific epilepsy syndromes in the affected relatives: (1) Case 3's maternal great uncle had unclassified epilepsy; (2) three third cousins of Case 9 had recurrent seizures in adulthood; (3) Case 14's grandmother had unprovoked seizures in adulthood, and this maternal grandmother's sister had febrile seizures in childhood; (4) Case 15's paternal grandfather's brother had seizures in childhood only; (5) a paternal first cousin of Case 16 had seizures, and there is also a history of febrile seizures in this patient's second cousins; and finally (6) Case 26's father had recurrent seizures attributed to a previous history of traumatic brain injury. Only one child (Case 10) presented with an inherited variant from an unaffected parent. Case 10's mother has no neurological manifestations, but is heterozygous for the variant c.862G $>$ A; p.Gly288Ser. Consanguinity was reported in four families from Saudi Arabia, Cases 5, 6, 7, and 8, and three of these four variants (c.1885A $>$ G, p.Lys629Glu; c.862G $>$ A, p.Gly288Ser; and c.2800G $>$ A; p.Ala934Thr) were found to be de novo. The parents of Case 6 were not available for testing (unknown inheritance of the variant c.1421G $>$ A; p.Arg474His).

Epilepsy of infancy with migrating focal seizures was the most common electroclinical syndrome, present in 18 patients $(66.7 \%, 18 / 27)$. Four children had an unclassified EOEE, three children were diagnosed with West syndrome, and two children were diagnosed with ADSHE at some point. Three children have had more than one electroclinical phenotype throughout their trajectories; Cases 3 and 4 initially presented with EIMFS evolving to West syndrome, whereas 
case 22's phenotype was initially consistent with unclassified EOEE, but the patient later developed ADSHE. Table 1 summarizes the demographics, relevant clinical information, KCNT1 variant details, and neuroimaging findings.

Focal impaired awareness motor (tonic or with tonic component) seizures were the most common seizure type (48.1\%, 13/27) observed in our cohort. In addition, a variety of other seizures were also observed, such as focal impaired awareness nonmotor (behavioral arrest, autonomic, emotional), focal impaired awareness motor (hyperkinetic, clonic), generalized tonic, generalized tonic-clonic, generalized myoclonic-tonic-clonic, generalized myoclonic, and generalized absence seizures (atypical). Estimated current seizure frequency reported by caregivers was markedly variable at the time of data collection, with three patients $(11.1 \%, 3 / 27)$ having $>500$ seizures/mo, nine patients $(33.4 \%, 9 / 27)$ having $>100$ up to 500 seizures/mo, three patients $(11.1 \%, 3 / 27)$ having $>50$ up to 100 seizures/mo, seven patients $(26 \%, 7 / 27)$ having 10 to 50 seizures/mo, and five patients $(18.5 \%, 5 / 27)$ having $<10$ seizures/mo. None of the caregivers reported seizure freedom.

The presence of developmental plateauing, EIMFS, and seizure burden were not associated with a specific genetic variant. Five children harboring c.2800G $>$ A; p.Ala934Thr were compared with the rest of the cohort with respect to the presence of developmental plateauing $(P=.13)$, phenotype consistent with EIMFS $(P=.48)$, and estimated seizure frequency $>500$ per month $(P=.22)$. In addition, four children harboring c.862G $>$ A; p.Gly288Ser were compared with the rest of the cohort with respect to the presence of developmental plateauing $(P=.93)$, phenotype consistent with EIMFS $(P=.44)$, and estimated seizure frequency $(P=.38)$. There were no significant differences between the genotypes analyzed compared with the rest of the cohort with respect to the aforementioned characteristics. The most common phenotype seen in our cohort consisted of nonambulatory $(92.3 \%, 24 / 26$; unavailable data in one patient), nonverbal $(88 \%, 22 / 25$; unavailable data in two patients), hypotonic (74\%, 20/27), and spastic $(48.1 \%, 13 / 27)$ patients, with acquired microcephaly (65.3\%, 17/26; unavailable data in one patient) and cortical visual impairment $(60 \%, 15 / 25$; unavailable data in two patients). Only five children $(18.5 \%, 5 / 27)$ were diagnosed with involuntary movements (see Table 1).

Brain MRI was abnormal in the majority of children $(59.3 \%, 16 / 27)$. The most common findings were delayed myelination $(68.7 \%, 11 / 16$; or $40.7 \%, 11 / 27$ of all patients) and thin corpus callosum $(43.7 \%, 7 / 16$; or $26 \%, 7 / 27$ of all patients), usually but not always accompanied by brain atrophy (37.5\%, 6/16; or $22 \%, 6 / 27$ of all patients). The presence of an abnormality in the brain MRI was further evaluated in the two most common genetic variants in comparison with the rest of the cohort. There was no correlation between the presence of an abnormality in patients with specific genotypes in comparison with the rest of the cohort (ie, c.2800G $>$ A; p.Ala934Thr vs other patients, $P=.97$; c.862G $>$ A; p.Gly288Ser vs other patients, $P=.68$ ).

Systemically, these children often had constipation (40.7\%, 11/27), gastroesophageal reflux disease $(33.4 \%$, $9 / 27$ ), and aspiration pneumonia $(33.4 \%, 9 / 27)$. Only one child (Case 7) had a cardiac malformation (ventricular septal defect) and supraventricular ectopic activity.

Table 2 shows the current antiepileptic drugs (AEDs), number of total AEDs tried for each patient, alternative therapies and estimated response for each, and final disposition for each patient. Twenty-three children are still under follow-up $(85.2 \%, 23 / 27)$, and four have passed away $(14.8 \%, 4 / 27)$. Cause of death was due to complications of systemic diseases $(n=1)$, progression of illness $(n=1)$, and redirection of the goal of care $(n=2)$. There were no reports of sudden unexpected death in epilepsy (SUDEP). Of the 23 children being followed, the number of current AEDs in use ranged from one to five $($ mean $=2.7, \mathrm{SD}=1.2$, median $=2$ ). Including the patients who died, the total number of AEDs tried ranged from three to $16($ mean $=7.4, \mathrm{SD}=3.1$, median $=7)$. Given the high number of medications tried in a relatively short period of time and the subjective nature of responses, along with a variety of different combinations, we were unable to identify one or two AEDs that were particularly effective in controlling seizures. Eleven patients were tried on quinidine, with doses ranging from 20 to $60 \mathrm{mg} / \mathrm{kg} / \mathrm{d}$ (mean $=35.9 \mathrm{mg} /$ $\mathrm{kg} / \mathrm{d}, \mathrm{SD}=11.1$, median $=40 \mathrm{mg} / \mathrm{kg} / \mathrm{d}$ ) and duration of therapy from 1 to 28 months (mean $=9.1$ months, $\mathrm{SD}=9.5$, median $=4$ months). As per caregivers, estimated response was marked or some improvement in five $(45.4 \%, 5 / 11)$, but there was no response whatsoever in six children $(54.6 \%, 6 / 11)$. Five children $(45.4 \%, 5 / 11)$ developed QTc prolongation while on quinidine, but none discontinued therapy for this reason. Irritability and vomiting were reported in one child each. The age at administration of quinidine ranged from 2 to 37 months (mean $=11.4$ months, $\mathrm{SD}=9.8$, median $=$ 8 months). There was no statistically significant correlation between responses to quinidine and the earlier age at onset of therapy $\left(r_{\mathrm{s}}=0.22917, P\right.$ [2-tailed $\left.]=.49\right)$.

CBD was used in seven patients $(26 \%, 7 / 27)$, with highly variable doses ranging from 0.25 to $10 \mathrm{mg} / \mathrm{kg} / \mathrm{d}$ (mean = $6 \mathrm{mg} / \mathrm{kg} / \mathrm{d}, \mathrm{SD}=4.4$, median $=7.5 \mathrm{mg} / \mathrm{kg} / \mathrm{d}$ ). Estimated response was reported as marked or some improvement in five children $(71.4 \%, 5 / 7)$ and minimal in two $(28.6 \%, 2 / 7)$. CBD was well tolerated in all patients, with no side effects reported by the caregivers. Diet therapies were tried in 14 patients $(51.8 \%, 14 / 27)$, which included the ketogenic diet in 12 and low glycemic index diet in two. Eight children $(57.2 \%, 8 / 14)$ had marked or some improvement, and six $(42.8 \%, 6 / 14)$ had minimal or no improvement. There was no statistically significant correlation when children harboring c.2800G $>$ A; p.Ala934Thr or c.862G $>$ A; p.Gly288Ser were compared 


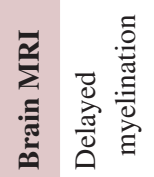

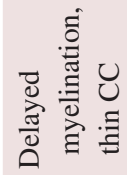

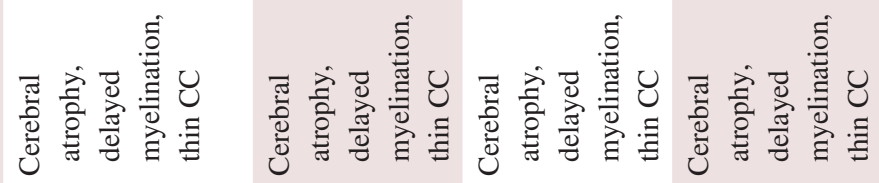

竞

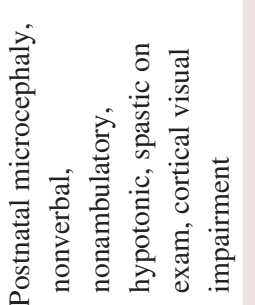
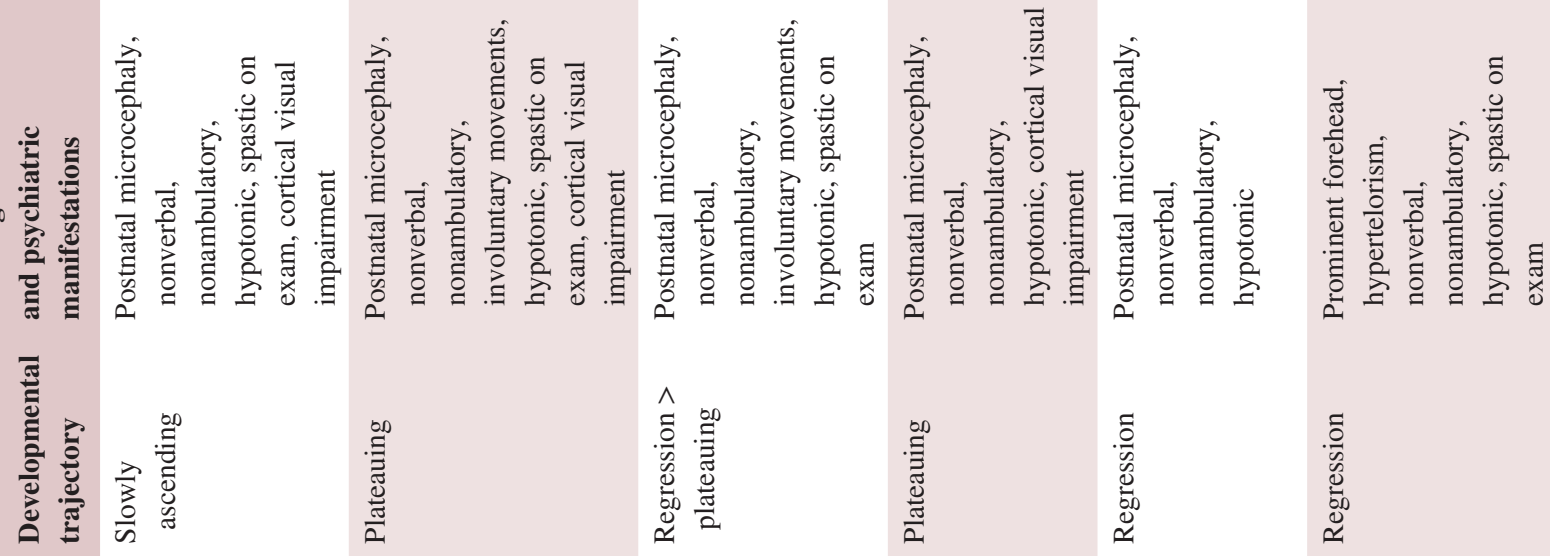

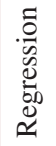

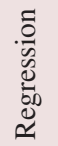

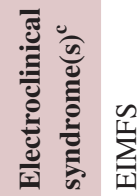

$\sum_{i=1}^{\infty}$

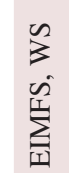

$\sum_{\text {III }}^{\infty}$

$\sum_{=1}^{\infty}$

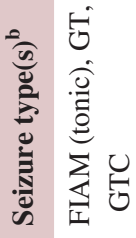

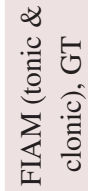

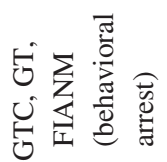

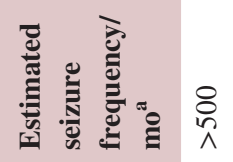

$\begin{array}{lll}8 & \stackrel{0}{n}\end{array}$

ט

$\sum_{j}^{U}$

บับ

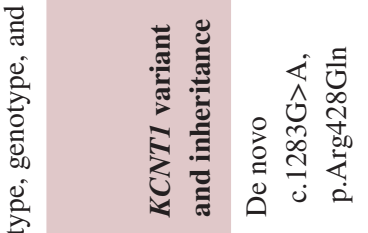

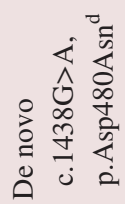

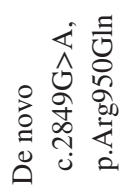

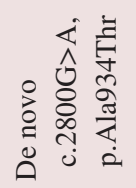

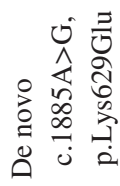

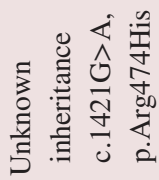

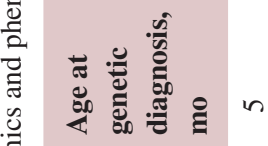

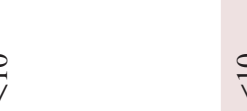

\begin{tabular}{l}
8 \\
$\stackrel{1}{1}$ \\
\hdashline
\end{tabular}

ถุ

है.

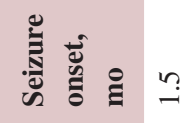

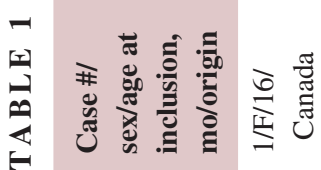

\丶

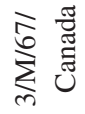

商苞

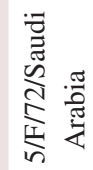

这语语 


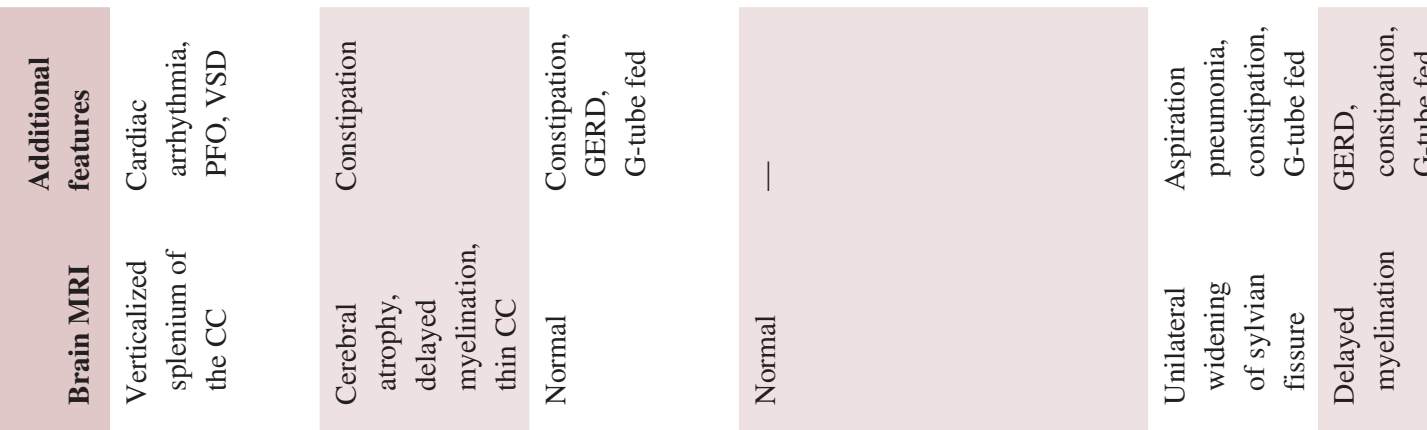

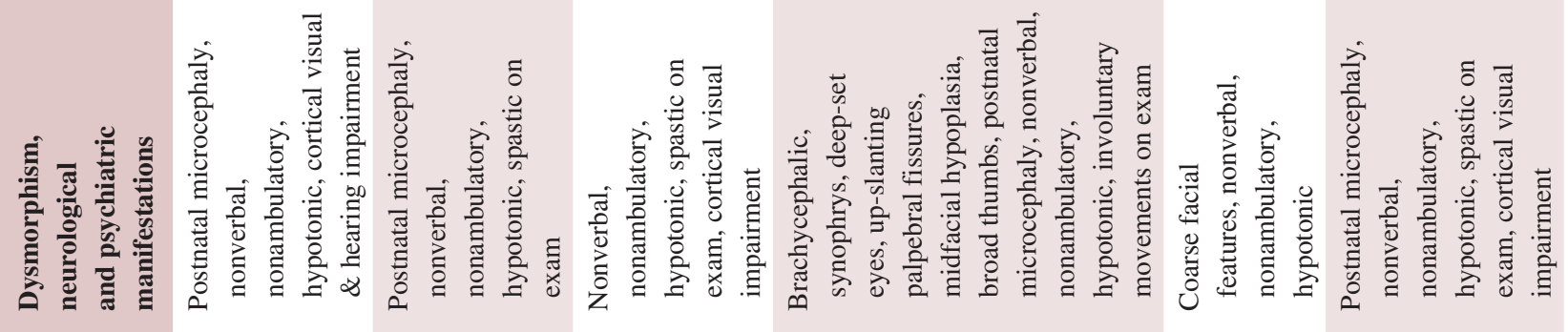

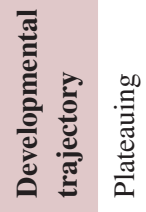

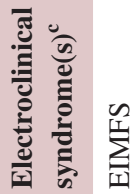

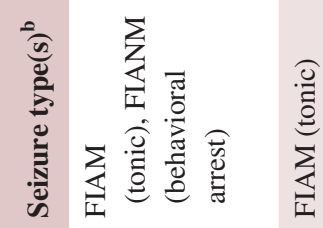

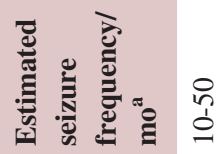

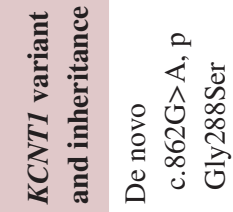

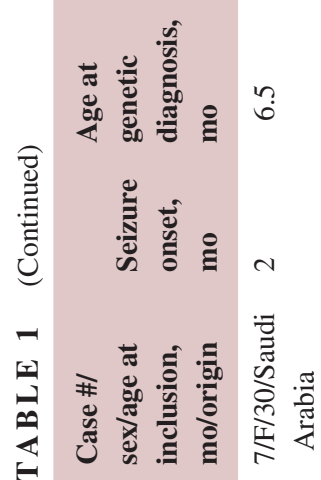

in

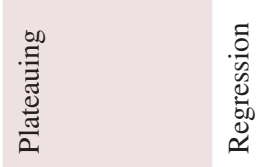

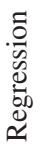

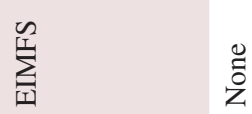

5

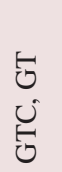

$\frac{8}{\frac{1}{n}}$

$\stackrel{\circ}{\mathrm{v}}$

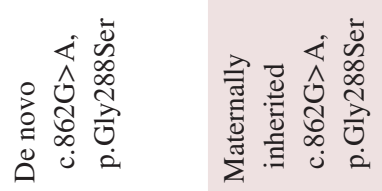

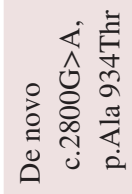

ก

6

蔐高

喜高

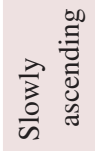

3

咅 v
8
0
0

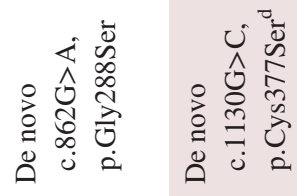

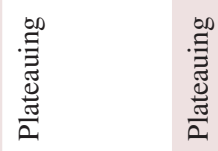

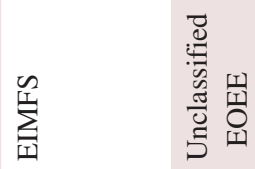

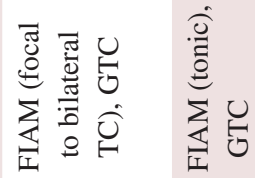

$\stackrel{8}{\frac{8}{6}}$

?

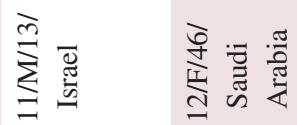




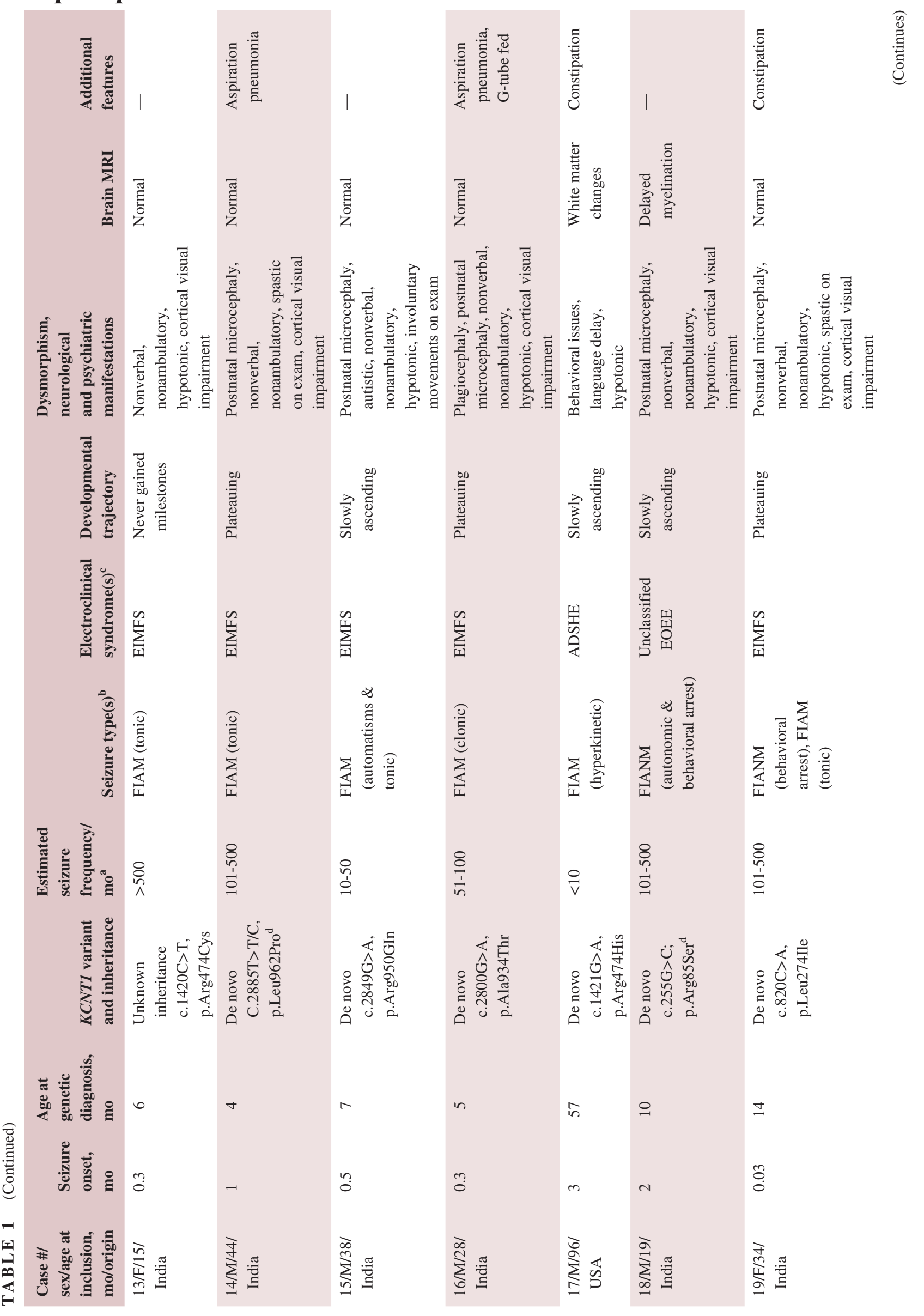




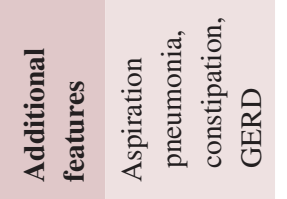

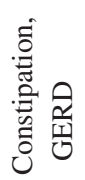

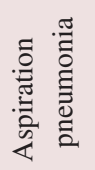

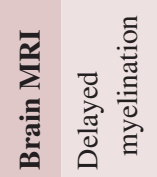

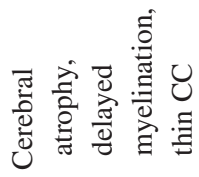

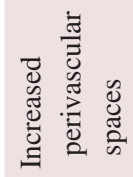

हี

ฮี
ปี
Z

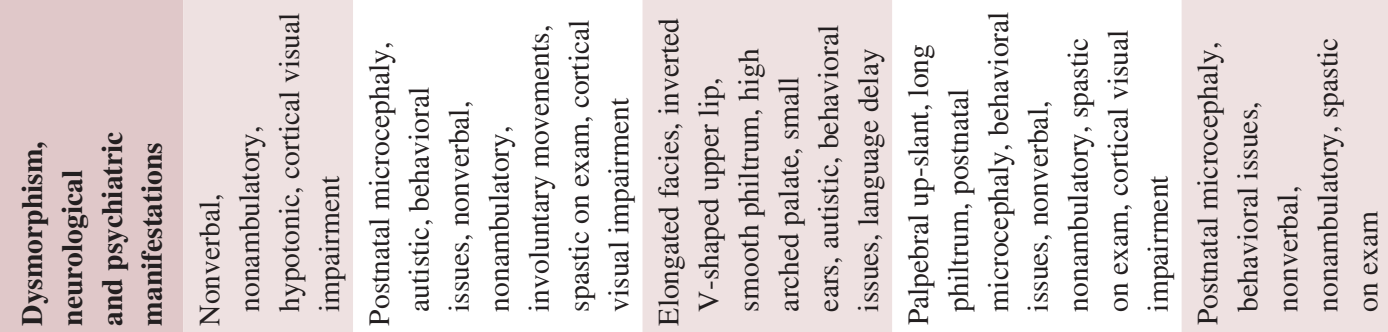
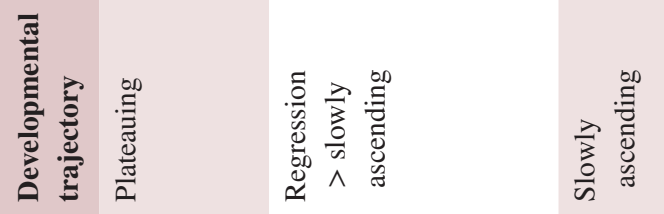

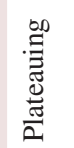

謌

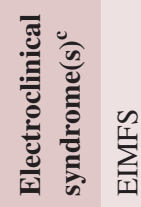

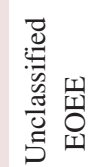

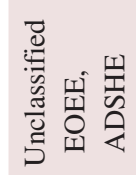

$\sum_{\text {III }}^{\infty}$

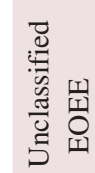

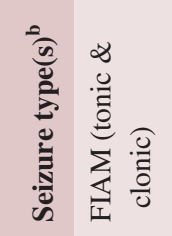

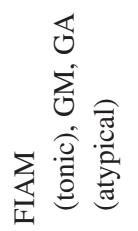

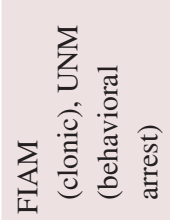

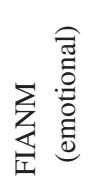

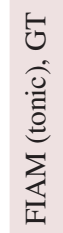

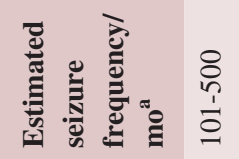

8
0
1
0

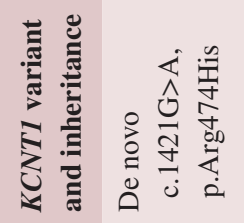

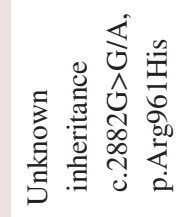

은

ํํำ

8
2
1
0

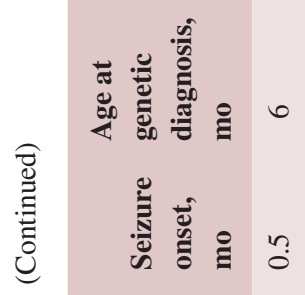

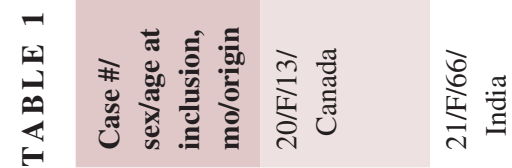
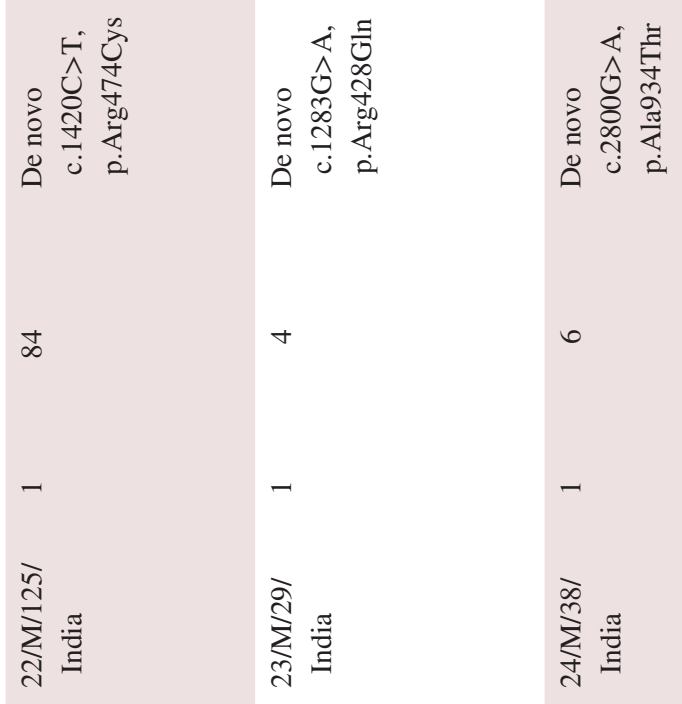

๑

章全 

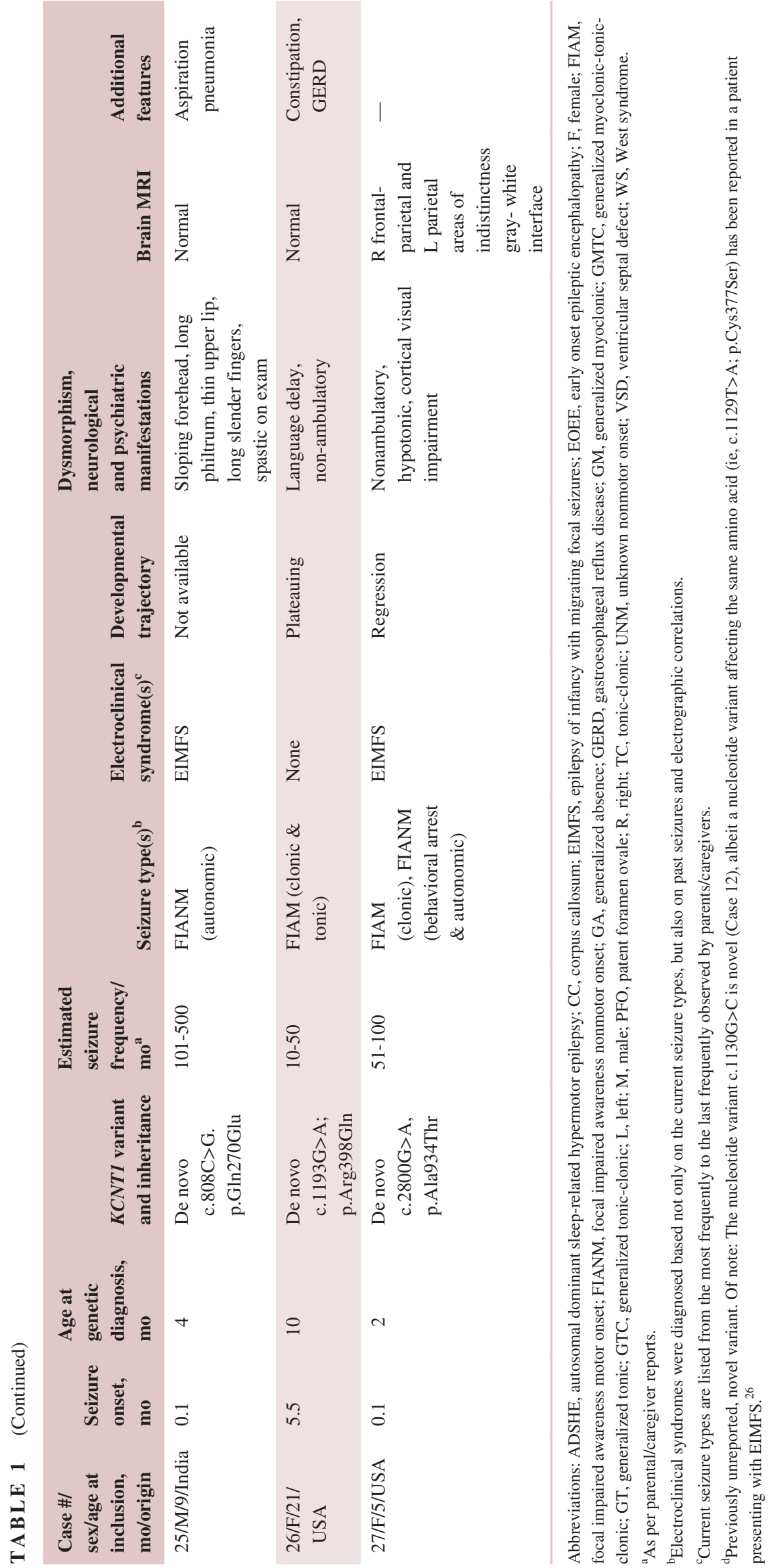
TA B L E 2 Current and past therapies, estimated response to alternative treatments, and final disposition

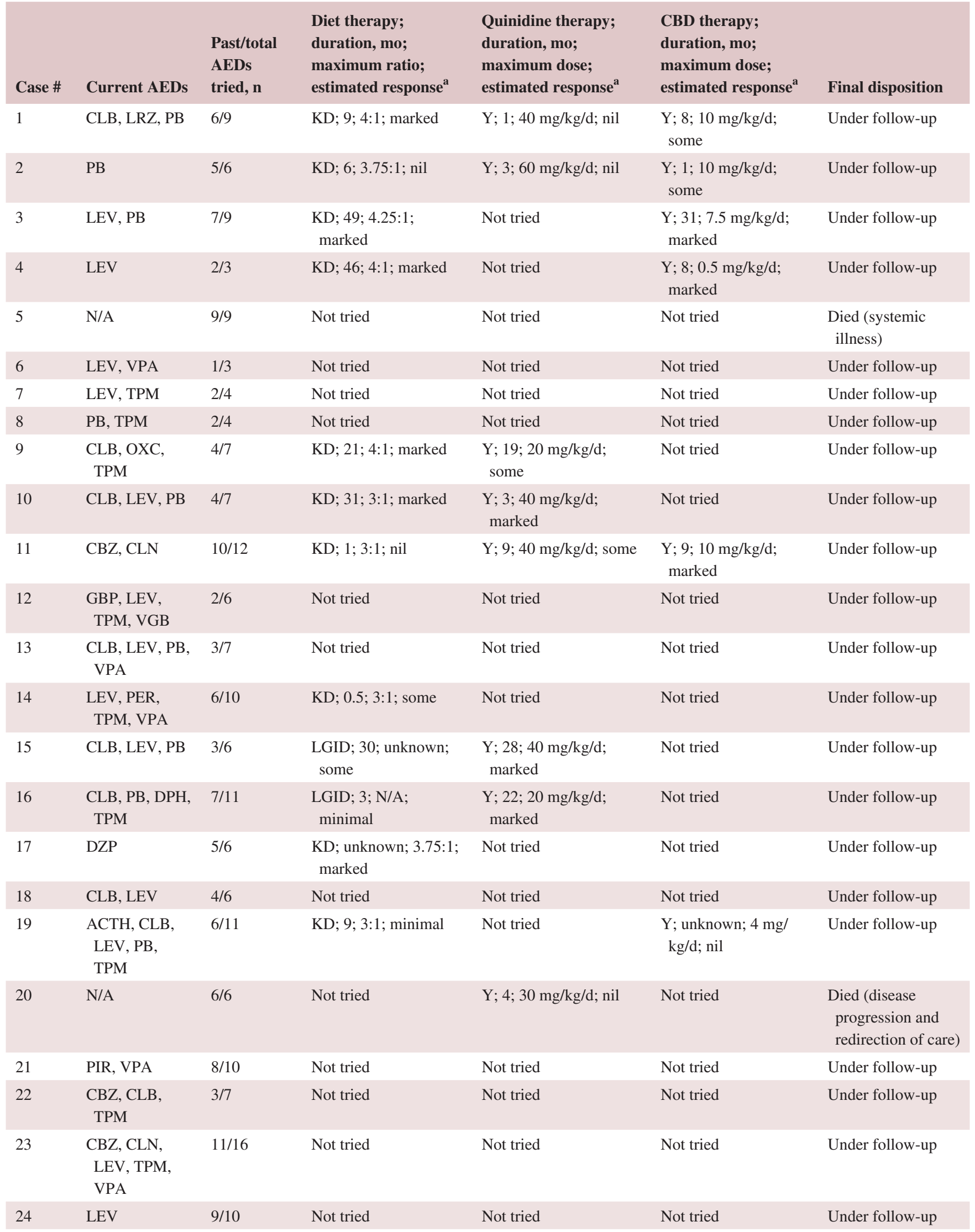


TABLE 2 (Continued)

\begin{tabular}{|c|c|c|c|c|c|c|}
\hline Case \# & Current AEDs & $\begin{array}{l}\text { Past/total } \\
\text { AEDs } \\
\text { tried, n }\end{array}$ & $\begin{array}{l}\text { Diet therapy; } \\
\text { duration, mo; } \\
\text { maximum ratio; } \\
\text { estimated response }^{\text {a }}\end{array}$ & $\begin{array}{l}\text { Quinidine therapy; } \\
\text { duration, mo; } \\
\text { maximum dose; } \\
\text { estimated response }^{\text {a }}\end{array}$ & $\begin{array}{l}\text { CBD therapy; } \\
\text { duration, mo; } \\
\text { maximum dose; } \\
\text { estimated response }^{\text {a }}\end{array}$ & Final disposition \\
\hline 25 & N/A & $11 / 11$ & $\mathrm{KD} ; 1 ; 3.5: 1 ;$ nil & $\mathrm{Y} ; 2 ; 35 \mathrm{mg} / \mathrm{kg} / \mathrm{d} ;$ nil & Not tried & $\begin{array}{l}\text { Died (systemic } \\
\text { illness) }\end{array}$ \\
\hline
\end{tabular}

Abbreviations: ACTH, adrenocorticotropic hormone; AED, antiepileptic drug; CBD, cannabidiol; CBZ, carbamazepine; CLB, clobazam; CLN, clonazepam; DPH, phenytoin; DZP, diazepam; GBP, gabapentin; KD, ketogenic diet (classical); LEV, levetiracetam; LIGD, low glycemic index diet; LRZ, lorazepam; N/A, not applicable; OXC, oxcarbazepine; PB, phenobarbital; PER, perampanel; PIR, piracetam; TPM, topiramate; VGB, vigabatrin; VPA, valproate; Y, yes.

${ }^{a}$ Estimated response scale: marked improvement, $>50 \%$ reduction in seizures; some improvement, $25 \%-50 \%$ reduction in seizures; minimal, $<25 \%$ reduction in seizures; nil, no change in seizure frequency.

with the rest of the cohort with respect to treatment response to quinidine, $\mathrm{CBD}$, or diet therapy.

\section{4 | DISCUSSION}

This observational study is one of the largest international cohorts examining pediatric patients diagnosed with KCNT1-related epilepsy. There was no selection bias with respect to the phenotypic characterization of this patient sample, given that the essential criterion was related to the presence of pathogenic or likely pathogenic KCNT1 variants. Some may argue that the presence of ADSHE could be underestimated due the cutoff age of the inclusion criteria, but we believe our data reflect the current circumstances of clinicians dealing with KCNT1-related epilepsy in infancy and childhood. The 17-month gap between first seizures (mean age at onset of seizures $=1.7$ months) and diagnosis of KCNT1-related epilepsy (mean age at diagnosis $=18.8$ months) in our cohort reflects the true odyssey for families and physicians alike.

As previously reported by different authors, KCNT1 pathogenic variants are highly pleiotropic and associated with a variety of phenotypes. ${ }^{8,18,27}$ In our study, two-thirds of patients presented with EIMFS; however, unclassified EOEE, West syndrome, and ADSHE were also present, similar to previous reports. Further analysis of recurrent variants and seizure burden related to specific genotypes produced no significant results, reinforcing the variability in gene expression and high pleiotropy of KCNT1 pathogenic variants.

However, our data obtained from 27 affected children enabled us to refine the phenotypic characterization, which may help in earlier recognition of these patients; early onset of tonic seizures that are medically refractory, with plateauing of milestones, hypotonia, cortical visual impairment, and acquired microcephaly should promptly raise a heightened index of suspicion for KCNT1-related epilepsy, likely manifesting as EIMFS or unclassified EOEE. Obviously, other seizure types and developmental trajectories can be seen. Similar to our results, the high prevalence of acquired microcephaly was also noted by Kuchenbuch et al to be as high as $90 \%$ after 3 years of follow-up. ${ }^{28}$ Given the cross-sectional nature of our study, we were not able to delineate different phases experienced by EIMFS patients over time, recently described as the stormy phase, stabilization period, and chronic phase. ${ }^{28}$

Somatic mosaicism (ie, DNA alteration occurring at the postzygotic stage) may also contribute to the phenotypic heterogeneity seen in some epilepsy genes. As previously demonstrated for "de novo" epileptic encephalopathies, $8.3 \%$ of parents have mosaicism of their child's pathogenic variant, particularly when there is parental history of seizures. ${ }^{29}$ Interestingly, the high prevalence of positive family history of seizures $(\sim 30 \%)$ in our patients and in another study ${ }^{28}$ might indicate the need for future research focused on relatives of patients with KCNT1-related epilepsy.

Although we often consider that neuroimaging is normal in the genetic epileptic encephalopathies, our data reinforce that in KCNT1-related epilepsies brain MRI can be abnormal (59\% of our children). From those with abnormal imaging, we found that delayed myelination, thin corpus callosum, and brain atrophy were the most common findings, albeit some of these abnormalities (ie, delayed myelination and brain atrophy) are likely to depend on whether the neuroimaging was obtained at earlier or later stages of the disease. One of our patients (Case 27) was found to have areas of indistinctness in the gray-white matter interface, which could suggest an underlying malformation of cortical development, as recently described in patients with $K C N T 1$ pathogenic mutations and ADSHE. $^{11}$

Given the expression of $K C N T 1$ in muscle tissue, gonads, and the pituitary gland, it has been proposed that $K C N T 1 \mathrm{mu}-$ tations can be involved in cardiac anomalies, SUDEP, and 
precocious puberty. ${ }^{5,27,28}$ One of our patients (Case 7) was diagnosed with a ventricular septal defect and supraventricular ectopic activity. However, unlike in the other reported cases, neither SUDEP nor precocious puberty was reported in our series, possibly due to limited long-term follow-up of this cohort. A cross-sectional study including older patients as well as long-term follow-up assessments could help identify precocious puberty and perhaps SUDEP if these conditions are related to KCNT1-related epilepsy.

Poor response to AEDs is a common characteristic among KCNT1-related epilepsy patients regardless of the phenotype. Not different from other studies, ${ }^{18,28}$ our patients were exposed to several AEDs over time $($ mean $=7.4, \mathrm{SD}=3.1)$, with no seizure freedom achieved. Moreover, more than onehalf $(55 \%, 15 / 27)$ reported $>50$ seizures monthly. These data promptly led clinicians and families to look for alternative treatments. After some case reports successfully reported treating KCNT1-related epilepsy with quinidine, ${ }^{12,13}$ several authors unfortunately have not been able to reproduce the same outcomes. ${ }^{14-17}$ In addition, a small blinded, placebo-controlled, crossover trial that included six ADSHE patients did not show a significant difference in seizure frequency during the quinidine phase compared with placebo, and paroxysmal arousals were similarly unchanged. There were no patients achieving the $50 \%$ reduction mark. ${ }^{30}$

In our retrospective analysis, nearly $50 \%$ of (5/11) patients had marked or some seizure reduction with quinidine. Twenty-seven percent (3/11) reported $>50 \%$ seizure reduction, and $18.1 \%$ (2/11) reported from $25 \%$ to $50 \%$ seizure reduction. Our findings were similar to those from the largest cohort of KCNT1-related epilepsy, which evaluated 20 patients taking quinidine and found nearly 50\% (9/20) of patients with some response, including $20 \%$ of patients having at least $50 \%$ seizure reduction. ${ }^{18}$ Neither seizure freedom nor worsening of seizures was reported in our patients. In addition, we were not able to establish statistical correlation suggesting an age-dependent response to quinidine, as previously reported in the literature. ${ }^{15}$

Other than quinidine, we were also able to record reasonable efficacy of diet therapy and CBD. When taking into consideration patients exposed to diet therapies, $43 \%$ $(6 / 14)$ had $>50 \%$ seizure reduction. Analyzing the response to the ketogenic diet (not including the low glycemic index diet), half of the patients (6/12) had $>50 \%$ seizure reduction. Reasonable response to the ketogenic diet has also been reported, with a response rate of $31 \%(9 / 29)$ as per caregivers and physicians. ${ }^{18}$ For those who responded well to $\mathrm{CBD}$, two of three were previously on the ketogenic diet, making any conclusions on the CBD efficacy merely speculative. In addition, the small number of patients on CBD and the lack of consistency of the dosage prescribed (from 0.25 to $10 \mathrm{mg} / \mathrm{kg} / \mathrm{d}$ ) limit our capacity to draw meaningful conclusions.
In addition to the limitations related to our study method, which include missing data and lack of longitudinal follow-up, our study is hampered by the limited number of patients for subset analysis. This is difficult to overcome when gathering data in a rare condition. Parental reports of seizure frequency (sometimes hard to recognize due to subtle manifestations, or sometimes overcalled due to the presence of abnormal movements and behavioral issues) and the lack of standardized use of some therapies (ie, CBD and quinidine doses) are further limitations of our study. Moreover, with our current data, it is still unclear whether somatic mutations are particularly relevant in KCNT1-related epilepsy. The low level of parental mosaicism could be underestimated given that the great majority of parental testing in our study was through Sanger sequencing and not through next generation sequencing analysis. ${ }^{31}$

In summary, through international collaboration and in comparison with previous literature data, we were able to delineate the common aspects within this highly pleiotropic entity, KCNT1-related epilepsy: early onset refractory tonic seizures, likely (but not exclusively) manifesting as EIMFS or unclassified EOEE, along with milestones plateauing, hypotonia, cortical visual impairment, and acquired microcephaly. Supportive but not mandatory neuroimaging findings included delayed myelination, thin corpus callosum, brain atrophy, and rarely malformations of cortical development. Despite the lack of satisfactory evidence, alternative treatments such as the ketogenic diet and quinidine seem to be well tolerated and may help achieve seizure reduction of $>50 \%$.

\section{ACKNOWLEDGMENTS}

We thank Dr Camila C. Henriques de Aquino for her valuable assistance with statistical analysis and interpretation.

\section{CONFLICT OF INTEREST}

K.P.V. was supported by an institutional seed grant for infantile epilepsy registry from the Amrita University. He was also supported by academic grants from the Kerala Association of Neurologists and the Indian Epilepsy Association, Kochi. None of the other authors has any conflict of interest to disclose. We confirm that we have read the Journal's position on issues involved in ethical publication and affirm that this report is consistent with those guidelines.

\section{ORCID}

Felippe Borlot (iD https://orcid.org/0000-0001-7897-4700

Majid Alfadhel (D) https://orcid.org/0000-0002-9427-7240

Ravindra Arya (D) https://orcid.org/0000-0003-0873-9718

Kenneth A. Myers (D) https://orcid.

org/0000-0001-7831-4593

Jitendra K. Sahu (D) https://orcid.org/0000-0001-5194-9951

Suvasini Sharma (D) https://orcid.org/0000-0002-3135-3306 


\section{REFERENCES}

1. Barcia G, Fleming MR, Deligniere A, et al. De novo gain-of-function KCNT1 channel mutations cause malignant migrating partial seizures of infancy. Nat Genet. 2012;44:1255-9.

2. Bhattacharjee A, Kaczmarek LK. For $\mathrm{K}+$ channels, $\mathrm{Na}+$ is the new Ca2+. Trends Neurosci. 2005;28:422-8.

3. Ruffin VA, Gu XQ, Zhou D, et al. The sodium-activated potassium channel Slack is modulated by hypercapnia and acidosis. Neuroscience. 2008;151:410-8.

4. Niday Z, Tzingounis AV. Potassium channel gain of function in epilepsy: an unresolved paradox. Neuroscientist. 2018;24:368-80.

5. The Human Protein Atlas. KCNT1. Available at: https://www.prote inatlas.org/ENSG00000107147-KCNT1/tissue. Accessed October 3, 2019.

6. Ishii A, Shioda M, Okumura A, et al. A recurrent KCNT1 mutation in two sporadic cases with malignant migrating partial seizures in infancy. Gene. 2013;531:467-71.

7. Heron SE, Smith KR, Bahlo M, et al. Missense mutations in the sodium-gated potassium channel gene KCNT1 cause severe autosomal dominant nocturnal frontal lobe epilepsy. Nat Genet. 2012;44:1188-90.

8. Ohba C, Kato M, Takahashi N, et al. De novo KCNT1 mutations in early-onset epileptic encephalopathy. Epilepsia. 2015;56:e121-8.

9. Hansen N, Widman G, Hattingen E, Elger CE, Kunz WS. Mesial temporal lobe epilepsy associated with KCNT1 mutation. Seizure. 2017;45:181-3.

10. Routier L, Verny F, Barcia G, et al. Exome sequencing findings in 27 patients with myoclonic-atonic epilepsy: is there a major genetic factor? Clin Genet. 2019;96:254-60.

11. Rubboli G, Plazzi G, Picard F, et al. Mild malformations of cortical development in sleep-related hypermotor epilepsy due to KCNT1 mutations. Ann Clin Transl Neurol. 2019;6:386-91.

12. Bearden D, Strong A, Ehnot J, DiGiovine M, Dlugos D, Goldberg EM. Targeted treatment of migrating partial seizures of infancy with quinidine. Ann Neurol. 2014;76:457-61.

13. Mikati MA, Jiang Y-H, Carboni M, et al. Quinidine in the treatment of KCNT1-positive epilepsies. Ann Neurol. 2015;78:995-9.

14. Chong PF, Nakamura R, Saitsu H, Matsumoto N, Kira R. Ineffective quinidine therapy in early onset epileptic encephalopathy with KCNT1 mutation. Ann Neurol. 2016;79:502-3.

15. Abdelnour E, Gallentine W, McDonald M, Sachdev M, Jiang Y-H, Mikati MA. Does age affect response to quinidine in patients with KCNT1 mutations? Report of three new cases and review of the literature. Seizure. 2018;55:1-3.

16. Dilena R, DiFrancesco JC, Soldovieri MV, et al. Early treatment with quinidine in 2 patients with epilepsy of infancy with migrating focal seizures (EIMFS) due to gain-of-function KCNT1 mutations: functional studies, clinical responses, and critical issues for personalized therapy. Neurotherapeutics. 2018;15:1112-26.

17. Numis AL, Nair U, Datta AN, et al. Lack of response to quinidine in KCNT1-related neonatal epilepsy. Epilepsia. 2018;59:1889-98.

18. Fitzgerald MP, Fiannacca M, Smith DM, et al. Treatment responsiveness in KCNT1-related epilepsy. Neurotherapeutics. 2019;16:848-57.
19. Baumer FM, Sheehan M. Quinidine-associated skin discoloration in KCNT1-associated pediatric epilepsy. Neurology. 2017;89:2212.

20. Richards S, Aziz N, Bale S, et al. Standards and guidelines for the interpretation of sequence variants: a joint consensus recommendation of the American College of Medical Genetics and Genomics and the Association for Molecular Pathology. Genet Med. 2015; 17:405-24.

21. Nykamp K, Anderson M, Powers M, et al. Sherloc: a comprehensive refinement of the ACMG-AMP variant classification criteria. Genet Med. 2017;19:1105-17.

22. Harris PA, Taylor R, Thielke R, Payne J, Gonzalez N, Conde JG. Research electronic data capture (REDCap) — a metadata-driven methodology and workflow process for providing translational research informatics support. J Biomed Inform. 2009;42:377-81.

23. Harris PA, Taylor R, Minor BL, et al. The REDCap consortium: building an international community of software platform partners. J Biomed Inform. 2019;95:103208.

24. Patil AA, Vinayan KP, Roy AG. Two south Indian children with KCNT1-related malignant migrating focal seizures of infancyclinical characteristics and outcome of targeted treatment with quinidine. Ann Indian Acad Neurol. 2019;22:311-5.

25. Mandaan P, Jauhari P, Gupta A, Chakrabarty B, Gulati S. A quinidine non responsive novel KCNT1 mutation in an Indian infant with epilepsy of infancy with migrating focal seizures. Brain Dev. 2018;40:229-32.

26. Kawasaki Y, Kuki I, Ehara E, et al. Three cases of KCNT1 mutations: malignant migrating partial seizures in infancy with massive systemic to pulmonary collateral arteries. J Pediatr. 2017;191:270-4.

27. Møller RS, Heron SE, Larsen LHG, et al. Mutations in KCNT1 cause a spectrum of focal epilepsies. Epilepsia. 2015;56:e114-20.

28. Kuchenbuch M, Barcia G, Chemaly N, et al. KCNT1 epilepsy with migrating focal seizures shows a temporal sequence with poor outcome, high mortality and SUDEP. Brain. 2019;142:2996-3008.

29. Myers CT, Hollingsworth G, Muir AM, et al. Parental mosaicism in "de novo" epileptic encephalopathies. N Engl J Med. 2018;378:1646-8.

30. Mullen SA, Carney PW, Roten A, et al. Precision therapy for epilepsy due to KCNT1 mutations: a randomized trial of oral quinidine. Neurology. 2018;90:e67-72.

31. Qin L, Wang J, Tian X, et al. Detection and quantification of mosaic mutations in disease genes by next-generation sequencing. J Mol Diagn. 2016;18:446-53.

How to cite this article: Borlot F, Abushama A, Morrison-Levy N, et al. KCNT1-related epilepsy: An international multicenter cohort of 27 pediatric cases. Epilepsia. 2020;61:679-692. https://doi.org/10.1111/ epi. 16480 